\title{
Search for CO gas in Pluto, Centaurs and Kuiper Belt objects at radio wavelengths
}

\author{
D. Bockelée-Morvan ${ }^{1}$, E. Lellouch ${ }^{1}$, N. Biver ${ }^{1}$, G. Paubert ${ }^{2}$, J. Bauer ${ }^{3}$, P. Colom ${ }^{1}$, and D. C. Lis ${ }^{4}$ \\ 1 Observatoire de Paris, 5 place Jules Janssen, 92195 Meudon Cedex, France \\ 2 Instituto de Radioastronomía Millimétrica, Avenida Divina Pastora 7, Núcleo Central, 18012 Granada, Spain \\ 3 Institute for Astronomy, University of Hawaii, 2680 Woodlawn Dr., Honolulu, HI 96822, USA \\ 4 Division of Physics, Mathematics and Astronomy, California Institute of Technology, MS 320-47, Pasadena, \\ CA 91125, USA
}

Received 13 June 2001 / Accepted 4 July 2001

\begin{abstract}
We have searched for several rotational lines of $\mathrm{CO}$ in the Pluto-Charon system, Centaurs (Chiron, Pholus, Nessus, Asbolus, Chariklo and 1998 SG35) and Kuiper Belt objects (1994 TB, 1996 TL66, 1996 TO66, 1996 TP66 and 1998 WH4). The observations were performed with the $30 \mathrm{~m}$ telescope of the Institut de Radioastronomie Millimétrique for Pluto/Charon, and with the James Clerk Maxwell Telescope and Caltech Submillimeter Observatory for Centaurs and Kuiper Belt objects. A tentative 4.5- $\sigma J(2-1)$ CO line is present in the Pluto/Charon spectrum, which requires further confirmation. Assuming that Charon does not contribute to the $\mathrm{CO}$ emission, an upper limit of $1.2 \%$ and $7 \%$ is obtained for the $\mathrm{CO} / \mathrm{N}_{2}$ mixing ratio in Pluto's atmosphere, using the atmospheric thermal structure derived from the Stansberry et al. (1994, Icarus 111, 503) and Strobel et al. (1996, Icarus 120, 266) models, respectively. These upper limits are more constraining (by more than a factor of 6) than the upper limits reported by Young et al. (2001, Icarus, in press) from near-IR spectroscopy. None of the Centaurs or Kuiper Belt objects (KBO) were detected in CO. The CO production rate upper limit obtained for Chiron $\left(3-5 \times 10^{27} \mathrm{~mol} \mathrm{~s}^{-1}\right)$ over 1998-2000 years is a factor of 10 lower than the CO production rate derived from the marginal CO detection obtained in June 1995 by Womack \& Stern (1999, Astron. Vestnik 33, 216 ), using same modelling of CO emission. Upper limits obtained for other Centaurs are typically $\sim 10^{28} \mathrm{~mol} \mathrm{~s}^{-1}$, and between 1 and $5 \times 10^{28} \mathrm{~mol} \mathrm{~s}^{-1}$ for the best observed KBOs. The comparison between these upper limits and the CO outgassing rates of comet C/1995 O1 (Hale-Bopp) measured at large distances from the Sun shows that Centaurs and KBOs underwent significant CO-devolatilization since their formation.
\end{abstract}

Key words. planets and satellites: Pluto - Kuiper Belt - minor planets, asteroids - radio lines: solar system

\section{Introduction}

Our view of the outer Solar System has changed dramatically in the last decade, with the discovery of a large number of bodies in orbit beyond Neptune (see Jewitt \& Luu 2000, for a review). These so-called Kuiper Belt objects (KBO) form three distinct dynamical classes: the Plutinos near the 3:2 mean-motion resonance with Neptune, with dynamical similarities with Pluto; the scattered objects (SKBO) with large, eccentric and inclined orbits; and the classical KBOs with modest eccentricities and semimajor axes $a \geq 42 \mathrm{AU}$. It is believed that the inner edge of the Kuiper Belt, gravitationally influenced by Neptune and

Send offprint requests to: D. Bockelée-Morvan, e-mail: dominique.bockelee@obspm.fr
Uranus, has supplied the Centaurs population (Levison \& Duncan 1997). Indeed, these objects, with semimajor axes $8 \mathrm{AU}<a<35 \mathrm{AU}$, between those of Jupiter and Neptune, have strongly chaotic and short-lived orbits, so that a source region from which the Centaurs' population is continuously replenished must exist. In turn, Centaurs can be a source of short-period comets (Levison \& Duncan 1997). At the time of writing, $\sim 400 \mathrm{KBOs}, \sim 40$ SKBOs and $\sim 30$ Centaurs have been catalogued.

The study of the compositional properties of KBOs and Centaurs is of strong interest because they are likely to consist of the most unaltered matter from the Solar Nebula. Photometric measurements show evidence for a wide dispersion in the colors of KBOs and Centaurs, from neutral to very red (e.g. Barucci et al. 2000a; 
Jewitt \& Luu 2000, for a review), with some data sets arguing for a bimodal color distribution (Tegler \& Romanishin 1998, 2000). The color diversity has been interpreted as due to diverse degrees of impact rejuvenation of the surface altered by cosmic rays (Luu \& Jewitt 1996). Near-infrared spectroscopy revealed water ice at the surface of the Centaurs 10199 Chariklo, 5145 Pholus, 2060 Chiron, and possibly 8405 Asbolus (Brown \& Koresko 1998; McBride et al. 1999; Cruikshank et al. 1998; Foster et al. 2001; Luu et al. 2000; Kern et al. 2000; Barucci et al. 2000b). The depth of the water absorption features varies among objects and with observational date. Methanol ice (or another light hydrocarbon or oxidized derivative) is seen in the near-infrared spectrum of Pholus (Cruikshank et al. 1998). Water ice absorption features were detected in the spectrum of the KBO 1996 TO66 (Brown et al. 1999). Spectra obtained in a few other Centaurs and KBOs show no evidence for ice signatures (e.g. Luu \& Jewitt 1998; Brown et al. 2000a, $2000 \mathrm{~b}$ ). This diversity is intriguing and, up to now, no correlation between surface and dynamical properties has been established. In addition, Chiron presents cometarylike activity, while 8405 Asbolus, which has similar color and orbital characteristics, does not. The detection of CO outgassing in 29P/Schwassmann-Wachmann 1 (Senay \& Jewitt 1994; Crovisier et al. 1995; Festou et al. 2001), a comet sometimes classified as a Centaur which orbits at $\sim 6 \mathrm{AU}$ from the Sun, and of $\mathrm{CO}$ and $\mathrm{CO}_{2}$ in the coma of comet C/1995 O1 (Hale-Bopp) at large distances from the Sun (Biver et al. 1999a; Biver et al. 1999c; Crovisier et al. 1997), shows that the outgassing of very volatile species is at the origin of distant activity. Evolutionary thermal models of Centaurs and KBOs, assuming that these bodies are made of different ices and dust, have been developed to investigate their differentiation and outgassing (Prialnik et al. 1995; Prialnik \& Podolak 1995; Capria et al. 2000; De Sanctis et al. 2000, 2001).

Pluto is the only transneptunian object for which a bound atmosphere has been positively identified. Observationally, most of our knowledge of Pluto's atmosphere was acquired from the 1988 stellar occultation (see e.g. Millis et al. 1993; Stansberry et al. 1994; Yelle \& Elliot 1997), which revealed a tenuous (several $\mu$ bar) atmosphere exhibiting a strong thermal inversion with the atmospheric temperature rising from $\sim 40 \mathrm{~K}$ to $\sim 100 \mathrm{~K}$ in the first $\sim 20 \mathrm{~km}$. The subsequent discovery in the near-infrared of $\mathrm{N}_{2}, \mathrm{CO}$, and $\mathrm{H}_{2} \mathrm{O}$ ices on Pluto's surface (Owen et al. 1993; Cruikshank et al. 1997) in addition to the already detected $\mathrm{CH}_{4}$ ice, measurements of their relative abundances, and considerations on vapor pressure equilibria, established that $\mathrm{N}_{2}$ is the major gas of Pluto's atmosphere and that $\mathrm{CH}_{4}$ and $\mathrm{CO}$ must be secondary compounds. From near-IR spectroscopy, Young et al. (1997) reported the detection of methane gas at the percent level, but this detection has not yet been confirmed. Regarding CO, Barnes (1993) reported an upper limit on the $\mathrm{CO} J(1-0)$ rotational emission, but this upper limit was not stringent enough to constrain the
CO abundance. More recently, from high-resolution spectroscopy at $2.3 \mu \mathrm{m}$, Young et al. (2001) obtained upper limits of $\left(1.2 \times 10^{21}-9.2 \times 10^{24}\right) \mathrm{mol} \mathrm{cm}^{-2}$ for the $\mathrm{CO}$ column density. The corresponding $\mathrm{CO} / \mathrm{N}_{2}$ mixing ratio limit is $0.06-0.23$ in the best case (i.e. for a $\mathrm{N}_{2}$ surface pressure of $58 \mu \mathrm{bar})$.

However, theoretical expectations suggest a much lower $\mathrm{CO} / \mathrm{N}_{2}$ mixing ratio in Pluto's atmosphere. Based on their estimate of the $\mathrm{CO}$ abundance in the ice phase $\left(0.5 \%\right.$ of $\left.\mathrm{N}_{2}\right)$, and assuming that the $\mathrm{N}_{2}-\mathrm{CO}-\mathrm{CH}_{4}$ system is ideal (i.e. applying Raoult's law), Owen et al. (1993) estimated $\mathrm{CO} / \mathrm{N}_{2}=(0.02-0.2) \%$ in Pluto's atmosphere for an equilibrium surface temperature in the $34-58 \mathrm{~K}$ range. Lellouch (1994) and Strobel et al. (1996) adopted similar values $(0.075 \%$ and $0.046 \%)$ in their thermal structure models of Pluto's atmosphere. With the lower CO abundance in the ice (0.1-0.2\%) inferred by Douté et al. (1999) from a refined analysis of the near-IR observations, the CO atmospheric abundance should be accordingly lower. We note, however, that applying the same approach to Triton, which has a $\mathrm{CO}$ abundance of $0.05 \%$ in the ice phase at $38 \mathrm{~K}$ (Quirico et al. 1999), leads to an expected atmospheric CO mixing ratio of $4 \times 10^{-5}$, significantly below the $(0.25-5) \times 10^{-3}$ value suggested by Strobel et al. (1996) from thermal structure modelling.

As demonstrated by Lellouch (1994) and Strobel et al. (1996), the CO abundance impacts the thermal structure of Pluto's atmosphere, especially at sub-microbar pressure levels, through efficient cooling in pure rotational lines. In what follows, we report our attempt to detect $\mathrm{CO}$ in two of these lines, with the increased sensitivity of the 30-m telescope of the Institut de Radioastronomie Millimétrique (IRAM). We also present upper limits on the CO outgassing in a few Centaurs and KBOs obtained from CO observations at the James Clerk Maxwell Telescope (JCMT) and Caltech Submillimeter Observatory (CSO).

\section{Observations}

\subsection{Pluto}

Pluto observations were performed with the IRAM 30-m telescope on April 20, 21, 24, and 25, 2000, approximately one month before the opposition (June 1, 2000) when Pluto was at $\Delta=29.5$ AU from the Earth and at $r_{\mathrm{h}}=30.26$ AU from the Sun. They benefitted from the new generation receivers available at the telescope, and of the versatility of the system, which now allows the use of 4 receivers at the same time. The A100, B100 receivers were tuned to the frequency of the $\mathrm{CO} J(1-0)$ line $(115.271 \mathrm{GHz})$, and the $\mathrm{A} 230$ and $\mathrm{B} 230$ receivers to that of $\mathrm{CO} J(2-1)(230.538 \mathrm{GHz})$. In order to provide the best sensitivity, the observations were made in the frequency-switch mode. The frequency throw was 6.5 or $7.5 \mathrm{MHz}$ at $230 \mathrm{GHz}$, and 6.5 or $14 \mathrm{MHz}$ at $115 \mathrm{GHz}$, depending on the date. The frequency throw for the $230 \mathrm{GHz}$ receivers was optimized in order to minimize baseline ripples and to avoid, as far as possible, the contamination 
Table 1. Pluto observations of CO at the IRAM 30-m telescope.

\begin{tabular}{llllcl}
\hline Date & Line & $\begin{array}{l}t_{\text {int }}^{a} \\
{[\mathrm{~min}]}\end{array}$ & $\begin{array}{l}\text { Res. } \\
{[\mathrm{kHz}]}\end{array}$ & $\begin{array}{l}\mathrm{rms}^{b} \\
{[\mathrm{mK}]}\end{array}$ & $\begin{array}{l}T_{\mathrm{B}} \mathrm{d} \nu^{c} \\
{[\mathrm{mK} \mathrm{MHz}]}\end{array}$ \\
\hline $00 / 04 / 24-25$ & $J(1-0)$ & 1230 & 79 & 8 & $<6.6^{d}$ \\
$00 / 04 / 20-25$ & $J(2-1)$ & 2090 & 79 & 10 & $18 \pm 4$ \\
\hline
\end{tabular}

${ }^{a}$ Total integration time on source.

${ }^{b} 1-\sigma$ rms noise in units of main beam brightness temperature at $79 \mathrm{kHz}$ spectral resolution.

${ }^{c}$ Line area between -1 and $+1 \mathrm{MHz}$ from line center, in units of main beam brightness temperature.

${ }^{d} 3-\sigma$ upper limit.

of the Pluto CO $J(2-1)$ line by the CO emission from an extended, uncatalogued galactic source $(\mathrm{RA}(\mathrm{J} 2000)=$ 16:47:48; Dec(J2000) = -12:05:00), close to Pluto. The spectra were acquired at a high spectral resolution (23 and $47 \mathrm{kHz}$ ), using the 2048-channel autocorrelator as backend. The total integration time on source was $2 \times 1045 \mathrm{~min}$ for $\mathrm{CO} J(2-1)$, taking into account the two receivers. Technical problems on April 20 made the CO $J(1-0)$ observations obtained on this date unusable. Data were acquired under good weather conditions. SSB system temperatures at transit $\left(\sim 40^{\circ}\right.$ elevation $)$ were $\sim 260 \mathrm{~K}$ and from 290 to $360 \mathrm{~K}$ in the $T_{\mathrm{A}}^{*}$ scale, for the $J(1-0)$ and $J(2-1)$ lines, respectively.

A summary of the observations is given in Table 1. Noise levels and line intensities are expressed in the main beam brightness temperature scale $\left(T_{\mathrm{B}}\right)$, using beam efficiencies derived from continuum observations of Uranus (Table 2) and forward efficiencies of 0.95 and 0.90 for the 115 and $230 \mathrm{GHz}$ lines, respectively.

The obtained spectra are quite satisfactory in terms of baselines; a second-order polynomial was used for their subtraction. On the other hand, the spectra are contaminated by several strong lines, namely the CO emission line from the above-mentioned galactic source, and their negative counterparts which are 2 times weaker and are artifacts resulting from the frequency-switch procedure.

In the $\mathrm{CO} J(2-1)$ spectrum, a negative ghost feature, due to the galactic $\mathrm{CO}$ emission, is present between +1 and $3.4 \mathrm{MHz}$ with respect to the expected position of the Pluto's CO line (Fig. 1). Since Pluto's CO $J(2-1)$ line is expected to be at most $\sim 1 \mathrm{MHz}$ broad, for most atmospheric models, it should be free of contamination (Fig. 1). This was checked in more detail by looking at the velocity extent of the galactic emission line for which the signal-to-noise ratio is higher. We do not see any hint of galactic emission or absorption above the $1-\sigma$ level in the velocity range which would correspond to -1 to $1 \mathrm{MHz}$ with respect to Pluto, and the red wing of the galactic line extends at most to $0.6 \mathrm{MHz}$. The spectrum shown in Fig. 1 and the line area integrated from -1 to $1 \mathrm{MHz}$ with respect to line center (Table 1 ) suggest a marginal detection of the $\mathrm{CO} J(2-1)$ line at the 4.5- $\sigma$ level in the Pluto-Charon system. As discussed above, galactic
Table 2. Characteristics of the telescopes: half-power beam widths $(H P B W)$ and beam efficiencies $\left(B_{\text {eff }}\right)$.

\begin{tabular}{llll}
\hline Telescope & $\begin{array}{l}\text { Frequency } \\
{[\mathrm{GHz}]}\end{array}$ & $\begin{array}{l}H P B W \\
\text { [arcsec] }\end{array}$ & $B_{\text {eff }}$ \\
\hline IRAM 30-m & 115 & 21.5 & 0.75 \\
& 230 & 10.6 & 0.52 \\
JCMT & 230 & 21.0 & 0.69 \\
& 345 & 13.6 & 0.62 \\
CSO & 230 & 31.0 & 0.65 \\
& 345 & 21.0 & 0.60 \\
\hline
\end{tabular}

spectral contamination is excluded. However, this marginal detection requires further confirmation and we regard this tentatively measured line area of $18 \mathrm{mK} \mathrm{MHz}$ as an upper limit.

The CO $J(1-0)$ spectra acquired on April 24 and 25 are not affected by ghost lines, in contrast to those obtained on April 21 with a different frequency throw. Therefore, we only consider these 24-25 April $J(1-0)$ spectra in Table 1 and the following discussion. There is no hint of detection of the $\mathrm{CO} J(1-0)$ line.

The 3- $\sigma$ sensitivity limits in flux units, after smoothing to the expected $2 \mathrm{MHz}$ Pluto CO linewidths, are 13 and $23 \mathrm{mJy}$ for the $J(1-0)$ and $J(2-1)$ lines, respectively. This is more than one order of magnitude below the previously published upper limits based on observations with the Haystack $37-\mathrm{m}$ and the National Radio Astronomy Observatory (NRAO) 12-m telescopes (Barnes 1993, 1996).

\subsection{Centaurs and KBOs}

Observations of Centaurs and Kuiper Belt objects were performed with the CSO 10.4-m dish and with the JCMT 15-m antenna, both in Hawaii. They cover the March 1998 to July 2000 period. These observations were often performed as backup observations to other programmes. As a result, they did not always have the benefit of excellent weather conditions. Opacities at $230 \mathrm{GHz}$ during the observations varied from 0.03 to 0.4 , with an average around 0.12. At JCMT, the observational mode was, most of the time, frequency-switch with throws of 8.1 or 16.2 MHz. Beam-switching with a beam-throw of $120^{\prime \prime}$ was used on a few occasions. CSO observations were performed in beam-switching mode. We used the high spectral resolution spectrometers offered at these telescopes: $100 \mathrm{kHz}$ at CSO, and 94 to $188 \mathrm{kHz}$ at JCMT. The beam efficiencies were measured several times, by observing Mars, Jupiter, Saturn, and Uranus (Table 2). A log of the observations is given in Table 3 . Depending on the weather, or on the availability of the receivers, the $\mathrm{CO} J(2-1)$ or $J(3-2)$ lines were observed. At JCMT, the B3 $345 \mathrm{GHz}$ receiver is equipped with two mixers, which permits us to observe two orthogonal polarizations. For one object (10199 Chariklo), this receiver was used in double side band mode with $\mathrm{HCN} J(4-3)$ at $354.505 \mathrm{GHz}$ in the 

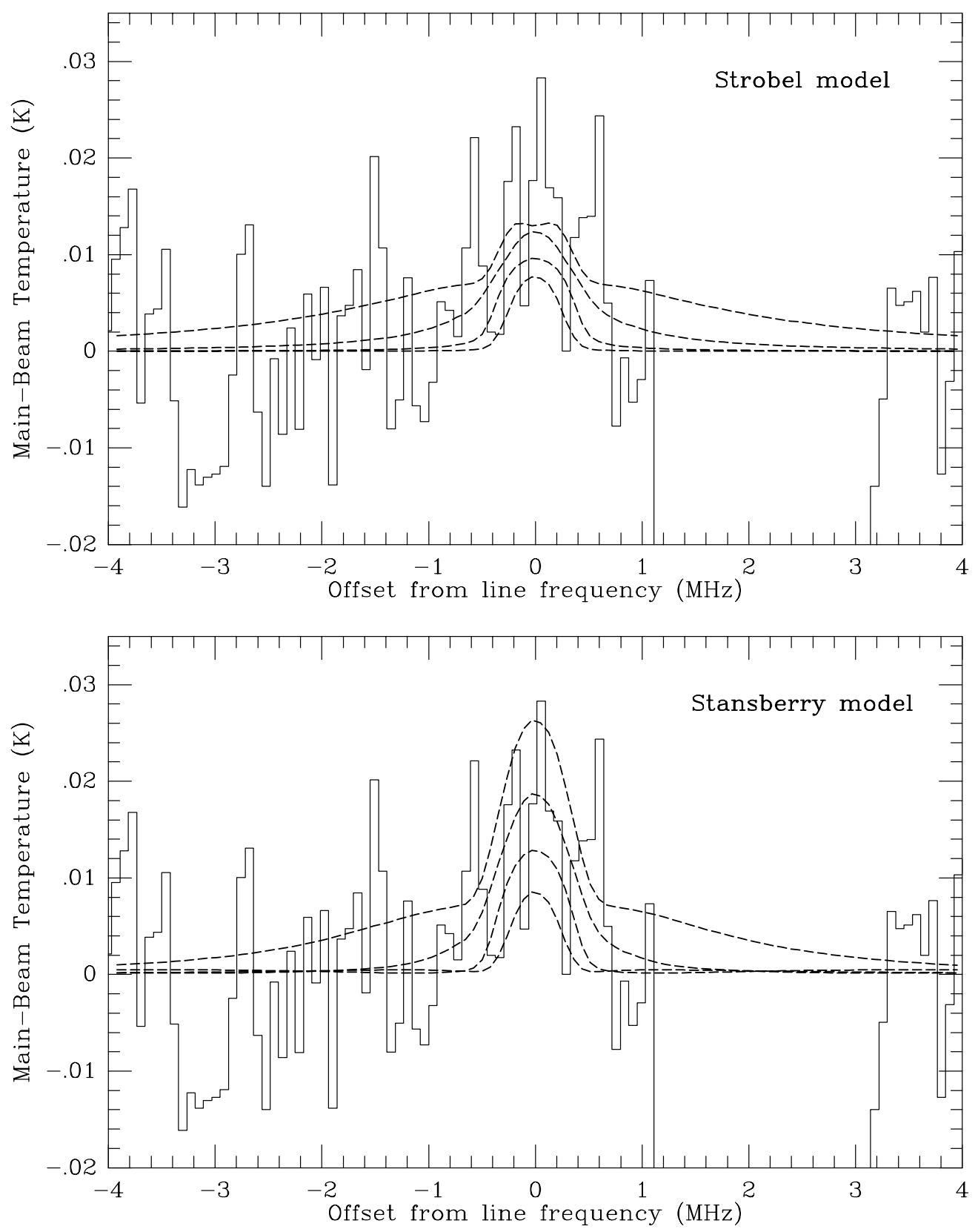

Fig. 1. Observed and modelled CO $J(2-1)$ Pluto spectra. Observed spectra are smoothed to $79 \mathrm{kHz}$ resolution. Top figure: synthetic spectra with the atmospheric thermal structure of Strobel et al. (1996) (solid lines in Fig. 2). Bottom figure: synthetic spectra with the atmospheric model of Stansberry et al. (1994) (dashed lines in Fig. 2). For each atmospheric model, synthetic spectra (dashed lines) are shown for CO mixing ratios of $0.01,0.1,1,10 \%$, from bottom to top.

upper side band and $\mathrm{CO} J(3-2)$ at $345.796 \mathrm{GHz}$ in the lower side band.

Six Centaurs and five KBOs were observed (Table 3), whose optical characteristics and diameters are summarized in Table 5. They were tracked using the most recent orbital elements available in the Minor Planet Circulars (MPC) or Minor Planet Electronic Circulars (MPEC). In the case of 1998 SG35 and 10199 Chariklo on 14-15 November 1998, improved elements showed that the ephemeris used for the observations was off by $\sim 7^{\prime \prime}$. Total beam offsets due to ephemeris and pointing uncertainties are given in Table 3 . None of the objects were detected in CO. The 3- $\sigma$ upper limits obtained for the integrated line intensity are typically $\sim 20 \mathrm{mK} \mathrm{km} \mathrm{s}^{-1}$ in the main beam brightness temperature scale, $T_{\mathrm{B}}$ (Table 3 ).

\section{Analysis}

\subsection{Pluto}

Although the telescope beam for the Pluto observations encompasses both Pluto and Charon, we assume that only Pluto contributes to the $\mathrm{CO}$ emission.

There is no clear observational evidence for the presence of an atmosphere around Charon. For a similar 
Table 3. Centaurs and KBOs observations.

\begin{tabular}{|c|c|c|c|c|c|c|c|c|c|c|}
\hline \multirow[t]{2}{*}{ Date } & \multirow[t]{2}{*}{$\begin{array}{l}r_{\mathrm{h}} \\
{[\mathrm{AU}]}\end{array}$} & \multirow[t]{2}{*}{$\begin{array}{l}\Delta \\
{[\mathrm{AU}]}\end{array}$} & \multirow[t]{2}{*}{ Tel. } & \multirow[t]{2}{*}{ Line } & \multirow[t]{2}{*}{$\begin{array}{l}t_{\text {int }}^{a} \\
{[\mathrm{~min}]}\end{array}$} & \multirow[t]{2}{*}{$\begin{array}{l}\text { offset }^{b} \\
{[\operatorname{arcsec}]}\end{array}$} & \multicolumn{2}{|c|}{$\begin{array}{l}T_{\mathrm{B}} \mathrm{d} v^{c} \\
{\left[\mathrm{mK} \mathrm{km} \mathrm{s}^{-1}\right]}\end{array}$} & \multirow[t]{2}{*}{$\begin{array}{c}(10 \mathrm{~K})^{d} \\
{\left[10^{28}\right.}\end{array}$} & \multirow[t]{2}{*}{$\begin{array}{l}Q(50 \mathrm{~K})^{d} \\
\left.\mathrm{~mol} \mathrm{~s}^{-1}\right]\end{array}$} \\
\hline & & & & & & & & & & \\
\hline $98 / 03 / 25.6$ & 8.9 & 8.2 & JCMT & $\mathrm{CO}(3-2)$ & 82 & 1.5 & $<21$ & & $<0.88$ & $<0.39$ \\
\hline $99 / 02 / 28.6$ & 9.3 & 9.2 & $\mathrm{CSO}$ & $\mathrm{CO}(3-2)$ & 64 & 2.5 & $<50$ & & $<3.3$ & $<1.5$ \\
\hline $99 / 06 / 09.5-11.5$ & 9.5 & 8.5 & JCMT & $\mathrm{CO}(2-1)$ & 314 & 2.0 & $<13$ & & $<0.55$ & $<0.57$ \\
\hline $99 / 06 / 24.4-27.4$ & 9.5 & 8.7 & $\mathrm{CSO}$ & $\mathrm{CO}(2-1)$ & 53 & 2.0 & $<48$ & $<34$ & $<2.2$ & $<2.2$ \\
\hline $00 / 07 / 26.4$ & 10.2 & 9.5 & $\mathrm{CSO}$ & $\mathrm{CO}(2-1)$ & 43 & 2.5 & $<49$ & & & \\
\hline 5145 Pholus & & & & & & & & & & \\
\hline $99 / 02 / 28.5$ & 13.9 & 13.3 & $\mathrm{CSO}$ & $\mathrm{CO}(3-2)$ & 85 & 3.0 & $<36$ & & $<4.6$ & $<1.7$ \\
\hline $99 / 06 / 24.3-27.3$ & 14.2 & 13.9 & $\mathrm{CSO}$ & $\mathrm{CO}(2-1)$ & 109 & 4.0 & $<33$ & & $<3.4$ & $<2.8$ \\
\hline $00 / 02 / 16.5-17.6$ & 14.8 & 14.5 & JCMT & $\mathrm{CO}(3-2)$ & 117 & 2.0 & $<44$ & & $<4.4$ & $<1.4$ \\
\hline $\begin{array}{l}7066 \text { Nessus } \\
99 / 06 / 24.4-25.5\end{array}$ & 15.0 & 14.0 & $\mathrm{CSO}$ & $\mathrm{CO}(2-1)$ & 53 & 4.0 & $<53$ & & $<5.6$ & $<4.6$ \\
\hline 8405 Asbolus & & & & & & & & & & \\
\hline $99 / 02 / 27.7-28.6$ & 9.2 & 8.8 & $\mathrm{CSO}$ & $\mathrm{CO}(3-2)$ & 43 & 4.5 & $<46$ & & $<3.0$ & $<1.4$ \\
\hline $99 / 06 / 26.3-28.3$ & 8.8 & 8.2 & $\mathrm{CSO}$ & $\mathrm{CO}(2-1)$ & 53 & 3.0 & $<42$ & & $<2.5$ & $<2.5$ \\
\hline 10199 Chariklo & & & & & & & & & & \\
\hline $98 / 11 / 14.6-15.7$ & 13.6 & 13.5 & $\mathrm{CSO}$ & $\mathrm{CO}(2-1)$ & 184 & 7.0 & $<21$ & & $<2.2$ & $<1.8$ \\
\hline $98 / 11 / 26.7$ & 13.5 & 13.3 & JCMT & $\mathrm{CO}(3-2)$ & 143 & 1.5 & $<21$ & & $<1.8$ & $<0.62$ \\
\hline & & & JCMT & $\mathrm{HCN}(4-3)$ & 143 & 1.5 & $<18$ & & $<0.78$ & $<0.04$ \\
\hline $99 / 02 / 28.4$ & 13.5 & 12.6 & CSO & $\mathrm{CO}(3-2)$ & 75 & 3.5 & $<40$ & $<31$ & $<3.6$ & $<1.3$ \\
\hline $00 / 01 / 02.5$ & 13.4 & 12.7 & $\mathrm{CSO}$ & $\mathrm{CO}(3-2)$ & 149 & 1.5 & $<58$ & & & \\
\hline $00 / 01 / 03.5-04.6$ & 13.4 & 12.7 & $\mathrm{CSO}$ & $\mathrm{CO}(2-1)$ & 192 & 2.5 & $<22$ & & $<2.0$ & $<1.7$ \\
\hline $\begin{array}{l}1994 \text { ТВ } \\
98 / 11 / 09.3-13.4\end{array}$ & 30.1 & 29.4 & $\mathrm{CSO}$ & $\mathrm{CO}(2-1)$ & 205 & 2.0 & $<30$ & & $<8.3$ & $<5.1$ \\
\hline 1996 TL66 & & & & & & & & & & \\
\hline $98 / 07 / 8.8-9.8$ & 35.1 & 35.5 & JCMT & $\mathrm{CO}(3-2)$ & 66 & 3.0 & $<27$ & $<15$ & $<6.8$ & $<1.4$ \\
\hline $98 / 10 / 31.44-4.5$ & 35.1 & 34.1 & JCMT & $\mathrm{CO}(3-2)$ & 153 & 2.0 & $<16$ & & & \\
\hline $99 / 08 / 22.5$ & 35.1 & 34.7 & JCMT & $\mathrm{CO}(3-2)$ & 29 & 2.0 & $<85$ & & & \\
\hline $98 / 11 / 09.4-10.5$ & 35.1 & 34.1 & $\mathrm{CSO}$ & $\mathrm{CO}(2-1)$ & 107 & 2.0 & $<47$ & $<16$ & $<5.7$ & $<3.3$ \\
\hline $00 / 07 / 26.6-31.6$ & 35.0 & 35.2 & $\mathrm{CSO}$ & $\mathrm{CO}(2-1)$ & 203 & 2.5 & $<17$ & & & \\
\hline 1996 ТО66 & & & & & & & & & & \\
\hline $99 / 08 / 22.6$ & 45.9 & 45.1 & JCMT & $\mathrm{CO}(3-2)$ & 30 & 1.5 & $<78$ & $<13$ & $<9.9$ & $<1.7$ \\
\hline $99 / 09 / 06.4-07.5$ & 46.0 & 45.0 & JCMT & $\mathrm{CO}(3-2)$ & 175 & 3.5 & $<28$ & & & \\
\hline $99 / 10 / 30.2-11 / 01.3$ & 46.0 & 45.2 & JCMT & $\mathrm{CO}(3-2)$ & 150 & 2.0 & $<21$ & & & \\
\hline $99 / 10 / 03.3-04.3$ & 46.0 & 45.0 & JCMT & $\mathrm{CO}(2-1)$ & 165 & 3.0 & $<21$ & & $<7.0$ & $<3.9$ \\
\hline $00 / 07 / 26.6$ & 46.0 & 45.5 & $\mathrm{CSO}$ & $\mathrm{CO}(2-1)$ & 43 & 2.0 & $<36$ & & $<19$ & $<10$ \\
\hline 1996 ТР66 & & & & & & & & & & \\
\hline $98 / 11 / 01.4-04.4$ & 26.4 & 25.4 & JCMT & $\mathrm{CO}(3-2)$ & 40 & 2.0 & $<17$ & & $<4.5$ & $<1.0$ \\
\hline $\begin{array}{l}1998 \text { WH24 } \\
00 / 01 / 3.4-4.5\end{array}$ & 42.4 & 41.7 & $\mathrm{CSO}$ & $\mathrm{CO}(2-1)$ & 59 & 2.5 & $<40$ & & $<19$ & $<10$ \\
\hline 1998 SG35 & & & & & & & & & & \\
\hline $98 / 10 / 31.4-11 / 01.4$ & 10.6 & 9.9 & JCMT & $\mathrm{CO}(3-2)$ & 68 & 7.5 & $<25$ & & $<1.8$ & $<0.77$ \\
\hline
\end{tabular}

${ }^{a}$ Total integration time: on+off source, in case of beam switching; on source, in case of frequency switching.

${ }^{b}$ Beam offsets due to ephemeris and pointing errors.

${ }^{c}$ Line area between -0.6 and $+0.6 \mathrm{~km} \mathrm{~s}^{-1}$ in units of main beam brightness temperature. 3- $\sigma$ upper limits are quoted. Values on the right are averages over several periods, as indicated by the vertical bars.

${ }^{d}$ Production rates upper limits computed with a kinetic temperature of either $T=10 \mathrm{~K}$ or $T=50 \mathrm{~K}$. Calculations take into account beam offsets.

atmospheric composition and structure, atmospheric escape per surface unit would be about five times larger at Charon than at Pluto (Trafton et al. 1988; Yelle \& Elliot 1997; Trafton et al. 1997). For atmospheres buffered by volatile ices, an integration of the escape fluxes over the Solar System age suggests a typical loss of $\mathrm{N}_{2}$ ice surface layers of $3 \mathrm{~km}$ for Pluto and $8 \mathrm{~km}$ for Charon. Therefore, in both cases, volatiles initially exposed on the surface are likely to be exhausted, but both bodies must have retained some of their original inventory. Whether an atmosphere is present now depends on resupply mechanisms. The presence of an atmosphere around Pluto and 
of volatile ices on its surface suggest that geological processes must operate on Pluto. Such processes, however, are less likely on Charon because of its small size, and the absence of volatile ice signatures from Charon's surface (see Cruikshank et al. 1997) suggests that Charon currently has no atmosphere. The possibility that Charon may capture some of Pluto's escaping atmosphere leads to a negligible, $10^{-7} \mu$ bar, pressure at Charon. Yet, we note that, from stellar occultation data, Elliot \& Young (1991) reported a hint of a charonian bound atmosphere with low scale height, implying the presence of heavy species (Ar, etc.). The reality of the detection is however not considered likely by Trafton et al. (1997). In any event, there is currently no support for the presence of CO in an hypothetical Charon atmosphere, justifying our assumption to attribute all of the observed signal to Pluto.

Modelling of the CO emission from Pluto was performed using a standard atmospheric radiative transfer code. Indeed, as shown by Strobel et al. (1996), the $\mathrm{CO}$ emission occurs in local thermodynamical equilibrium conditions down to pressure levels of about $10^{-5} \mu \mathrm{bar}$. This is 11.7 scale heights above the $1.2 \mu$ bar, $1250 \mathrm{~km}$ radius reference level indicated by the stellar occultation (Yelle \& Elliot 1997). For an upper atmospheric temperature in the range $80-106 \mathrm{~K}$, this corresponds to a $900-1400 \mathrm{~km}$ altitude above the $1250 \mathrm{~km}$ radius.

Because there remains considerable uncertainty about Pluto's atmospheric thermal structure, we used two different atmospheric models, both of which assume hydrostatic equilibrium. The first one is based on the thermal models of Strobel et al. (1996). These models account for solar heating in the near-infrared bands of $\mathrm{CH}_{4}$, and cooling in the $7.6 \mu \mathrm{m} \mathrm{CH}$ band and in the $\mathrm{CO}$ rotational lines. Strobel et al. investigated the sensitivity of their calculations to the surface pressure and $\mathrm{CH}_{4}$ abundance and vertical distribution. We specifically adopted the model with a $3 \mu$ bar surface pressure, in which most of the $\mathrm{CH}_{4}$ is confined to the bottom two scale heights (see their Fig. 11). This model reproduces the major features indicated by the stellar occultation data, namely a $15 \mathrm{~K} \mathrm{~km}^{-1}$ temperature gradient at the surface and a $\sim 100 \mathrm{~K}$ quasi-isothermal temperature just above the $p=2 \mu$ bar level. Yet, it implies a value of $\sim 1200 \mathrm{~km}$ for Pluto's surface radius, distinctly different from the radius indicated by the mutual event data (about $1158 \mathrm{~km}$, Buie et al. 1992). For this reason, Stansberry et al. (1994) suggested that Pluto may possess a $\sim 40 \mathrm{~km}$ deep "hidden" troposphere. They formulated a suite of models based on various tropospheric lapse rates and a Bates profile for the thermosphere. We adopted, as an alternative model, their profile with a tropospheric $\mathrm{d} T / \mathrm{d} z=-0.7 \mathrm{~K} \mathrm{~km} \mathrm{~s}^{-1}$ lapse rate, and a surface pressure of $24 \mu \mathrm{bar}$. This profile becomes isothermal at $106 \mathrm{~K}, \sim 45 \mathrm{~km}$ above Pluto's surface.

In both cases, the models were extended up to the $p=10^{-5} \mu$ bar level, which corresponds to $980 \mathrm{~km}$ and $1520 \mathrm{~km}$ altitudes, for the "Strobel" and "Stansberry" models, respectively. The two models are shown in Fig. 2 with the distance from Pluto's center as the altitude
Table 4. CO $J(2-1)$ line area within $\pm 1 \mathrm{MHz}$ from line center for different models and $\mathrm{CO} / \mathrm{N}_{2}$ mixing ratios.

\begin{tabular}{lcc}
\hline \multirow{2}{*}{ Mixing ratio } & \multicolumn{2}{c}{ Line area $[\mathrm{mK} \mathrm{MHz}]$} \\
\cline { 2 - 3 } & Strobel model & Stansberry model \\
\hline $10^{-4}$ & 4.13 & 4.73 \\
$10^{-3}$ & 7.59 & 9.02 \\
$10^{-2}$ & 13.2 & 17.0 \\
$10^{-1}$ & 18.2 & 27.1 \\
\hline
\end{tabular}

variable. We verified that the hydrodynamically escaping $\mathrm{CO}$ atmosphere has a negligible contribution to the CO line emission. This was done using the cometary model presented in Sect. 3.2 (see the related discussion). The only difference in the modelling with respect to Centaurs and KBOs is that collisional excitation is from $\mathrm{CO}-\mathrm{N}_{2}$ impacts instead of CO-CO impacts. With $v_{\text {exp }}=0.2 \mathrm{~km} \mathrm{~s}^{-1}, T=60 \mathrm{~K}$ at the exobase, a $\mathrm{N}_{2}$ escape rate of $2 \times 10^{27} \mathrm{~mol} \mathrm{~s}^{-1}$ (e.g. Krasnopolsky 1999) and $\mathrm{CO} / \mathrm{N}_{2}=1 \%$, the contribution of the escaping atmosphere to the $\mathrm{CO} J(2-1)$ line intensity is $0.05 \mathrm{mK} \mathrm{MHz}$.

In all models, $\mathrm{CO}$, which has the same mass as $\mathrm{N}_{2}$ and is photochemically stable, was assigned a vertically uniform distribution, and the CO mixing ratio was varied from $0.01 \%$ to $10 \%$. Pluto's brightness surface temperature was taken to be $31.5 \mathrm{~K}$, in agreement with bolometric measurements in the same wavelength range (Altenhoff et al. 1988; Lellouch et al. 2000).

Line opacities were calculated using Voigt profiles, using broadening coefficients ( $\mathrm{CO}$ by $\mathrm{N}_{2}$ ) from Colmont \& Monnanteuil (1986) and Semmoud-Monnanteuil \& Colmont (1987). The transfer equation was integrated over all emission angles. As Pluto's atmosphere has considerable extent compared to the planet's radius, limb emission was taken into account. Because the CO lines are heavily saturated already in vertical viewing, they remain optically thick in horizontal viewing up to large altitudes. For example, in the case of the Stansberry model and for $\mathrm{CO} / \mathrm{N}_{2}=0.1 \%$, the $\tau=1$ level in horizontal viewing for the $\mathrm{CO} J(2-1)$ line is reached at $p=2$ nanobar, i.e. $450 \mathrm{~km}$ above Pluto's surface. This gives an additional $106 \mathrm{~K}$ emitting area essentially equal to Pluto's solid area. Therefore, the brightness temperature contrast in the line core is boosted from a maximum of $75 \mathrm{~K}$ for the vertical viewing to an actual value of $\sim 190 \mathrm{~K}$ !

Figure 1 shows the comparison between observations and models for the CO $J(2-1)$ line. Calculated line areas are given in Table 4 . The effect of saturation is clearly evident, with the line contrast growing by only a factor of $2-3$ for a $\mathrm{CO}$ abundance increasing by three orders of magnitude. The tentatively measured area of $18 \mathrm{mK} \mathrm{MHz}$ and the spectrum appearance is reproduced for $\mathrm{CO} / \mathrm{N}_{2}=7 \%$, in the case of the Strobel model, and $\mathrm{CO} / \mathrm{N}_{2}=1.2 \%$ for the Stansberry model, but, as mentioned above, we regard these values as upper limits (note that, taking instead the $3-\sigma$ upper limit on the line area of $11.7 \mathrm{mK} \mathrm{MHz}$, the upper 

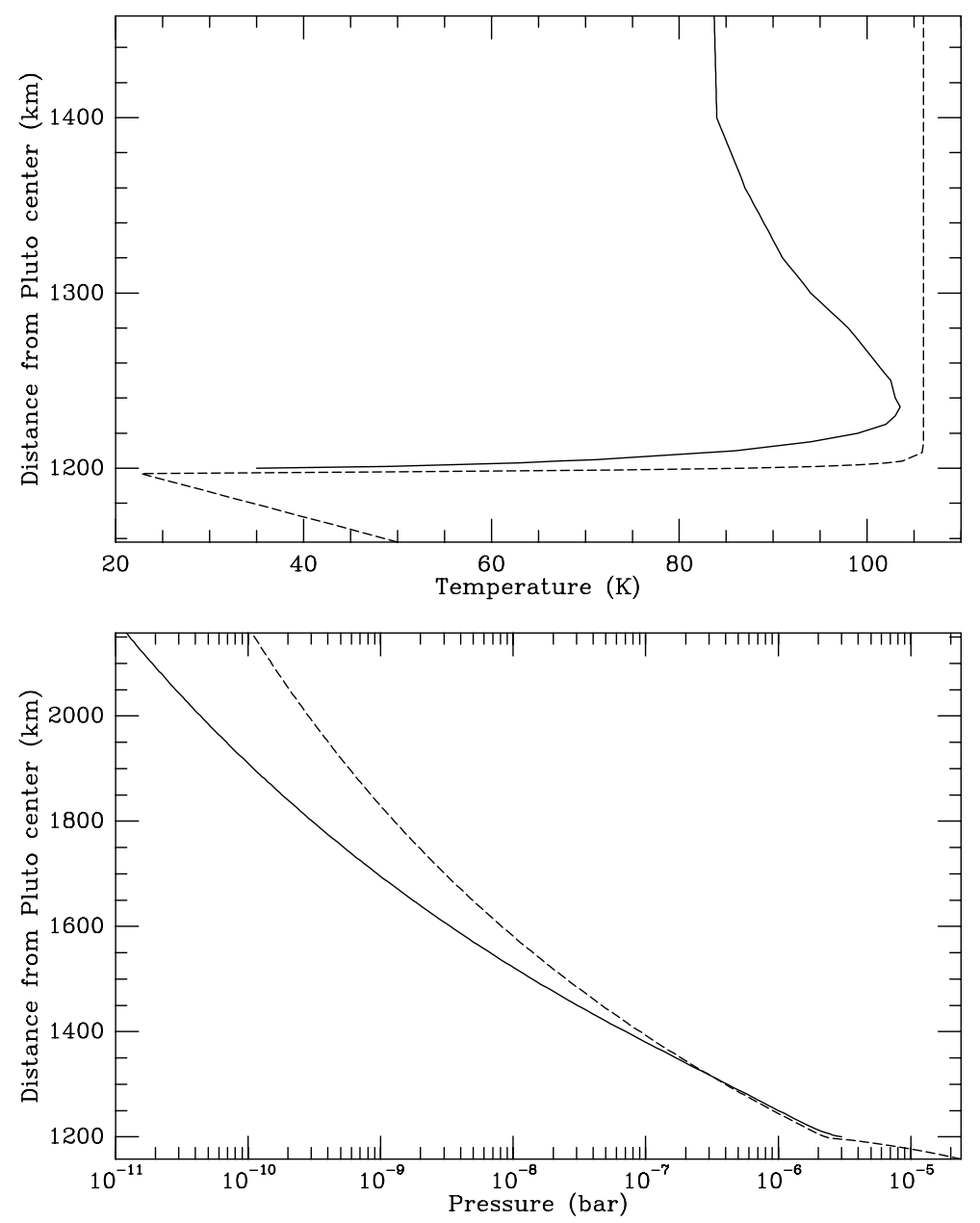

Fig. 2. The pressure-temperature-altitude thermal profiles adopted for the Pluto models. Solid line: "Strobel" model. Dashed line: "Stansberry" model. Since the two models correspond to different surface pressure and radius, the altitudes are expressed in terms of the distance from Pluto's center.

limits would be $\mathrm{CO} / \mathrm{N}_{2}<0.6 \%$ and $0.3 \%$, for the Strobel and Stansberry models, respectively). The higher sensitivity for the Stansberry model is due to the higher upper atmosphere temperature than in the Strobel model, which also causes it to be more extended. The possible presence of a troposphere and the exact value of the surface pressure are, in contrast, inconsequential to first order. We note that, with these values, the $\mathrm{CO} J(1-0)$ line shows a contrast of $\sim 0.003 \mathrm{mK}$ for both models and an integrated area of 3.1 (respectively 2.0) mK MHz for the Strobel (respectively Stansberry) model. This is fully consistent with the upper limit for this line given in Table 1. Essentially, the $115.271 \mathrm{GHz}$ line does not appear to be constraining because the filling factor of Pluto in the telescope main beam is four times smaller than at $230.538 \mathrm{GHz}$.

While our observations constrain the $\mathrm{CO}$ mixing ratio in Pluto's atmosphere rather than the integrated abundances, the above upper limits correspond to CO column densities of $1.2 \times 10^{20} \mathrm{~mol} \mathrm{~cm}^{-2}$ for the Strobel model and $8.75 \times 10^{19} \mathrm{~mol} \mathrm{~cm}^{-2}$ for the Stansberry model. For a "Stansberry-type" model with a $58 \mu$ bar surface pressure, the upper limit on the column density would be $\sim 2 \times 10^{20} \mathrm{~mol} \mathrm{~cm}^{-2}$, significantly more constraining than the $(1.2-3.5) \times 10^{21} \mathrm{~mol} \mathrm{~cm}^{-2}$ values reported by Young et al. (2001). We note also that the Young et al. observations do not provide any useful constraint in the case of a "Strobel" $3 \mu$ bar atmosphere.

Yet, our improved upper limits of $(1.2-7 \%)$ remain clearly insufficient for further understanding of Pluto's thermal structure and surface-atmosphere interaction. As mentioned in the introduction, the expected atmospheric $\mathrm{CO}$ mixing ratio for an ideal solid solution of $\mathrm{CO}$ in $\mathrm{N}_{2}$ is at least an order of magnitude less than our upper limits. As shown by Young et al. (2001), even if a "detailed balance" model is envisaged (Trafton et al. 1998), the CO atmospheric abundance cannot exceed (0.1-0.5)\%. The only situation that our upper limits can exclude is the case of isolated $\mathrm{CO}$ patches at temperatures similar or higher than $\mathrm{N}_{2}$ ice, which would dictate $\mathrm{CO} / \mathrm{N}_{2}$ mixing ratios of $5-20 \%$ for $T=35-50 \mathrm{~K}$. This situation is unlikely because 
Table 5. Objects characteristics.

\begin{tabular}{|c|c|c|c|c|c|}
\hline Designation & Name & & Type & $H^{a}$ & $D^{b}[\mathrm{~km}]$ \\
\hline $1977 \mathrm{UB}$ & 2060 & Chiron & Centaur & 6.5 & $168-180$ \\
\hline $1992 \mathrm{AD}$ & 5145 & Pholus & Centaur & 7.0 & 189 \\
\hline 1993 HA2 & 7066 & Nessus & Centaur & 9.6 & 80 \\
\hline 1995 GO & 8405 & Asbolus & Centaur & 9.0 & 106 \\
\hline 1997 CU26 & 10199 & Chariklo & Centaur & 6.4 & 275-302 \\
\hline 1998 SG35 & & & Centaur & 11.3 & 37 \\
\hline 1994 TB & 15820 & & $\mathrm{KBO}$ & 7.1 & 254 \\
\hline 1996 TL66 & 15874 & & SKBO & 5.4 & 555 \\
\hline 1996 TO66 & 19308 & & $\mathrm{KBO}$ & 4.5 & 840 \\
\hline 1996 ТР66 & 15875 & & $\mathrm{KBO}$ & 6.8 & 290 \\
\hline 1998 WH24 & 19521 & & $\mathrm{KBO}$ & 4.9 & 700 \\
\hline
\end{tabular}

${ }^{a}$ Absolute magnitude from Central Bureau for Astronomical Telegrams.

${ }^{b}$ References for diameters: Chiron (Bus et al. 1996; Groussin et al. 2000; Campins et al. 1994; Altenhoff \& Stumpff 1995); Pholus (Davies et al. 1993); Chariklo (Jewitt \& Kalas 1998; Altenhoff et al. 2001); for other objects, they were calculated from optical photometry alone, assuming a geometrical albedo of 0.04 .

$\mathrm{CO}$ and $\mathrm{N}_{2}$ ices are miscible in all proportions and have only a weak difference in volatility, which must strongly inhibit a CO segregation.

\subsection{Centaurs and KBOs}

CO observations of Centaurs and KBOs, and the single observation of HCN in 10199 Chariklo (Table 3), were interpreted using models developed for cometary atmospheres. These models assume freely escaping gas from the surface at constant velocity, namely a Haser density distribution:

$n(r)=\frac{Q}{4 \pi v_{\exp } r^{2}} \mathrm{e}^{-r / v_{\exp } \tau}$

where $Q\left[\mathrm{~mol} \mathrm{~s}^{-1}\right]$ is the total production rate $\left(Q_{\mathrm{CO}}\right.$ for $\mathrm{CO}), v_{\exp }$ is the gas expansion velocity, $\tau$ is the photodissociation lifetime $\left(\sim 1.5 \times 10^{6} \mathrm{~s}\right.$ and $\sim 6.3 \times 10^{4} \mathrm{~s}$ for $\mathrm{CO}$ and $\mathrm{HCN}$, respectively, at $\left.r_{\mathrm{h}}=1 \mathrm{AU}\right)$, and $r$ is the distance to body's center. This means that we neglect the possibility of a semi-captive atmosphere retained by gravity. This assumption may be questioned for the largest observed KBOs whose diameters $(D)$ reach $800 \mathrm{~km}$ (Table 5), but is probably reasonable given the situation at Charon, whose diameter is $\sim 1200 \mathrm{~km}$ (see Sect. 3.1 ). No models for the vertical structure of a putative atmosphere of Charon, and a fortiori of any other KBO, are available. In any event, CO line contrasts from hypothetical optically thick bound atmospheres around KBOs, if present, should be smaller than those for Pluto. Since the observational upper limits obtained on KBOs are less stringent than on Pluto, bound atmospheres should contribute at a negligible level to the observed upper limits (Table 3). Should any bound atmosphere be present, the computed production rate upper limits might be interpreted as upper limits on atmospheric CO escape rates.

Conservatively, we have assumed an expansion velocity $v_{\exp }=0.4 \mathrm{~km} \mathrm{~s}^{-1}$ for all objects, regardless of their distance to the Sun. CO outflow velocities $\sim 0.4-0.5 \mathrm{~km} \mathrm{~s}^{-1}$ have been measured in comets HaleBopp and 29P/Schwassmann-Wachmann 1 (P/SW1) at $r_{\mathrm{h}}>6 \mathrm{AU}$ (Crovisier et al. 1995; Biver et al. 1999a). This value of $0.4 \mathrm{~km} \mathrm{~s}^{-1}$ is higher, by a factor of a few (2-4 for the observed objects, assuming an albedo of 0.04), than the initial sonic gas velocity expected near the surface for freely escaping gas. This might account for the increase of the radial gas velocity during adiabatic expansion, although, for tenuous atmospheres, the small extent of the collision zone limits gas acceleration. For weakly bound atmospheres with hydrodynamically escaping gas, the sonic level is predicted to lie below the exobase (Chamberlain \& Hunten 1987). Therefore, terminal outflow velocities of a fraction of kilometer per second might be also expected. This is also the case for Pluto's atmosphere. Modelling shows that its escaping atmosphere does not fit completely the hydrodynamic case. At the exobase, $1500-4000 \mathrm{~km}$ altitude above the surface according to Krasnopolsky (1999), the gas radial velocity is subsonic and estimated to $\sim 1-2 \mathrm{~ms}^{-1}$ only (Krasnopolsky 1999; see also Trafton et al. 1997). However, due to the geometric effect (Hodges 1990), the transverse velocity due to random thermal motions at the exobase (where $T \sim 60 \mathrm{~K}$; Krasnopolsky 1999) will be converted at larger distances, in the collisionless atmosphere, to a radial velocity component. Taking into account that $3-\sigma$ upper limits on the line areas vary as the square root of the velocity window where they are computed, production rates upper limits scale proportionally to $v_{\mathrm{exp}}^{3 / 2}$. Possibly, in most cases, the $Q_{\mathrm{CO}}$ upper limits given in Tables 3 and 6 may be somewhat too conservative.

The computation of production rates upper limits requires the modelling of the $\mathrm{CO}$ rotational population distribution. We used a $\mathrm{CO}$ excitation model developed for cometary atmospheres, which includes collisional excitation by CO-CO impacts and radiative excitation by the Sun and $3 \mathrm{~K}$ cosmic background radiation (Biver et al. 1999b, and references therein). This model computes CO rotational populations as function of distance to object's center. An important parameter for CO excitation in distant objects is the gas kinetic temperature. Indeed, although the radial extent of the collisional region at thermal equilibrium is small compared to that of the region sampled by the beam, fluorescence equilibrium does not apply for the relevant rotational levels because of their long radiative lifetimes. In Table 3 , we present calculations performed with $T=10 \mathrm{~K}$ and $T=50 \mathrm{~K}$. Kinetic temperatures $\sim 10 \mathrm{~K}$ were measured in comets $\mathrm{P} / \mathrm{SW} 1$ and Hale-Bopp at $r_{\mathrm{h}}>6 \mathrm{AU}$ (Crovisier et al. 1995; Biver et al. 1999a), in good agreement with fast adiabatic cooling (Crifo et al. 1999). We explore a temperature of $50 \mathrm{~K}$, because expansion cooling can be "frozen" out in 
Table 6. Centaurs and KBOs: CO production rates upper limits averaging all data and $\mathrm{CO}$ depletion with respect to comet Hale-Bopp.

\begin{tabular}{llc}
\hline Object & $\begin{array}{l}Q_{\mathrm{CO}}^{a} \\
{\left[10^{28} \mathrm{~mol} \mathrm{~s}^{-1}\right]}\end{array}$ & $\begin{array}{c}\text { minimum CO }^{b} \\
\text { depletion }^{b}\end{array}$ \\
\hline 2060 Chiron & $<0.31-0.45$ & $38-55$ \\
5145 Pholus & $<1.0-2.3$ & $4-9$ \\
7066 Nessus & $<4.6-5.6$ & \\
8405 Asbolus & $<1.2-1.9$ & $4-6$ \\
10199 Chariklo & $<0.51-1.1$ & $20-43$ \\
1998 SG35 & $<0.77-1.8$ & \\
1994 TB & $<5.1-8.3$ & \\
1996 TL66 & $<1.3-4.4$ & $3-10$ \\
1996 TO66 & $<1.5-5.5$ & $3-11$ \\
1996 TP66 & $<1.0-4.5$ & $1-6$ \\
1998 WH24 & $<10-19$ & \\
\hline
\end{tabular}

${ }^{a} 3$ - $\sigma$ upper limits inferred with $T=50 \mathrm{~K}$ (lower values) and $T=10 \mathrm{~K}$ (larger values).

${ }^{b}$ Minimum CO depletion with respect to comet Hale-Bopp, assuming $Q_{\mathrm{CO}} \propto r_{\mathrm{h}}^{-2} \times D^{2}$, and using $D=40 \mathrm{~km}$ (Altenhoff et al. 1999) and $Q_{\mathrm{CO}}=10^{28} \mathrm{mols}^{-1}$ at $r_{\mathrm{h}}=9 \mathrm{AU}$ for comet Hale-Bopp (Biver et al. 1999c). Only depletions greater than 1 are given.

tenuous atmospheres and we cannot exclude significant heating linked to gravity (e.g. heating by dust in case of Chiron, Boice et al. 1993, or from near-IR $\mathrm{CH}_{4}$ bands in the extreme case of Pluto's atmosphere).

Table 3 presents $3-\sigma$ upper limits obtained on the $\mathrm{CO}$ and $\mathrm{HCN}$ production rates. Data acquired on the same line and with the same telescope, but at different dates, have been averaged. The final $3-\sigma Q_{\mathrm{CO}}$ upper limits, obtained by combining upper limits from different lines and telescopes, are given in Table 6. These upper limits are typically $\sim 10^{28} \mathrm{~mol} \mathrm{~s}^{-1}$ for Centaurs, and between 1 and $5 \times 10^{28} \mathrm{~mol} \mathrm{~s}^{-1}$ for the best observed KBOs. The upper limit obtained for the HCN production rate in 10199 Chariklo is strongly model dependent and is equal to $8 \times 10^{27} \mathrm{mols}^{-1}$ for $T=10 \mathrm{~K}$, and $4 \times 10^{26} \mathrm{~mol} \mathrm{~s}^{-1}$ for $T=50 \mathrm{~K}$.

Because Chiron presents cometary-like activity, rotational lines of $\mathrm{CO}$ were searched for in this Centaur on several occasions. Womack \& Stern (1999) announced the detection of the $J(1-0)$ line in June 1995, using the NRAO $12-\mathrm{m}$ telescope. With a pure thermal model at $T=10 \mathrm{~K}$ and $v_{\text {exp }}=0.2 \mathrm{kms}^{-1}$, assuming that $\mathrm{CO}$ emission fills the telescope beam, they inferred a $\mathrm{CO}$ production rate of $(1.5 \pm 0.8) \times 10^{28} \mathrm{~mol} \mathrm{~s}^{-1}$. The production rate we derive from their observations using our model with $v_{\exp }=$ $0.4 \mathrm{~km} \mathrm{~s}^{-1}$ is $2.9 \times 10^{28} \mathrm{~mol} \mathrm{~s}^{-1}$ and $5.2 \times 10^{28} \mathrm{~mol} \mathrm{~s}^{-1}$, for $T=10$ and $50 \mathrm{~K}$, respectively. Boice et al. (1999) observed the $J(1-0)$ line in February 1998 with the Nobeyama 45$\mathrm{m}$ telescope, from which we derive $Q_{\mathrm{CO}}<5 \times 10^{28} \mathrm{~mol} \mathrm{~s}^{-1}$ for $T=10 \mathrm{~K}$. Rauer et al. (1997) observed both the $J(1-0)$ and $J(2-1)$ lines with the IRAM 30-m in June, September and November 1995 and used the same model as ours for their interpretation. Their $3-\sigma$ upper limit obtained in June 1995 is consistent with the Womack and Stern's result, but the upper limit derived from the combined June 1995 to November 1995 data $\left(1 \times 10^{28} \mathrm{~mol} \mathrm{~s}^{-1}\right.$ for $T=10 \mathrm{~K}$ ) is clearly below the Womack \& Stern's value. Our derived upper limit of $\sim 0.3-0.5 \times 10^{28} \mathrm{~mol} \mathrm{~s}^{-1}$ for 1998-2000 (Table 6) is even more stringent, a factor of $\sim 10$ lower than the production rate derived from Womack and Stern's marginal detection using same modelling. From optical photometry, Chiron's activity shows short-term variability and a long-term trend to be lower near perihelion (February 1996) than near the aphelion (Bus et al. 2001; Lazzaro et al. 1997). Photometric measurements obtained in March, May 1995 (Bauer et al. 1997), January 1998, May 1999 and June 2000 (Bauer, private communication) show that Chiron was fainter over the years 1998-2000 by 0.3 magnitude (in absolute magnitude units) when compared to 1995 . This 0.3 magnitude variation would correspond to a $\mathrm{CO}$ production rate variation of $40 \%$ only, using the correlation between CO production rates and heliocentric magnitudes established from comet Hale-Bopp data (Biver 2001). Yet, the absolute $V$ magnitudes deduced from the 1998 to 2000 observations, $\left(H_{\mathrm{v}} \sim 7\right.$, not corrected from phase function; Bauer, private communication) indicate that Chiron was in its most quiescent state since its discovery. Therefore, we cannot exclude that Chiron was in outburst in June 1995, but, without any additional evidence for that, the low CO production rates derived for the June-September 1995 (Rauer et al. 1997) and 1998-2000 periods make the proposed detection of Womack and Stern at best tentative.

Thermal models of the interiors of Centaurs and KBOs have been developed to follow their evolution and differentiation and better understand their relationships with short-period comets (Capria et al. 2000; De Sanctis et al. 2000; De Sanctis et al. 2001). These models assume that these objects are porous bodies made of ices of different volatilities (amorphous $\mathrm{H}_{2} \mathrm{O}, \mathrm{CO}$ and $\mathrm{CO}_{2}$ as main constituents) and dust. They treat the heat diffusion in the porous material, the sublimation of volatile ices, the amorphous/crystalline phase transition, the diffusion of the produced gases and their recondensation or their escape into space. The model developed by De Sanctis et al. (2001) for KBOs includes the combined effects of radiogenic and solar heating. They show that the upper layers of Kuiper Belt objects could be strongly volatile-depleted, with CO ice completely absent down to several kilometers below the surface. CO production rates are expected to be very low (less than $10^{23} \mathrm{mols}^{-1}$ for an object of 200 km diameter; De Sanctis et al. 2000), making our upper limits meaningless in that context. However, the results of these simulations are strongly dependent upon size and model parameters, such as porosity and thermal conductivity, and more investigations of the thermal differentiation of KBOs are needed. Other simulations predict, for large objects, a runaway increase of their internal temperature due to radiogenic heating, which can squeeze out volatiles trapped in water amorphous ice and 
concentrate them near the surface where the temperature is lower (Haruyama et al. 1993; Prialnik \& Podolak 1995). Impacts may play also an important role in redistributing materials towards the surface, or ablating CO depleted layers. Transient cometary activity around 1996 TO66 has been proposed to explain a strong change of its lightcurve between September 1997 and September 1998 (Hainaut et al. 2000).

From their study of the thermal evolution of 2060 Chiron, Capria et al. (2000) concluded that Chiron's activity, if driven by $\mathrm{CO}$, can be explained only if $\mathrm{CO}$ is present near the surface as an ice, or as a gas trapped in the amorphous water ice. It is believed that Chiron originated from the Kuiper Belt and moved to its present orbit by gravitational perturbations. If the upper layers underwent CO-ice devolatization in the Kuiper Belt, as it follows from the model of De Sanctis et al. (2001), then Chiron's activity could be explained by the release of trapped CO during the amorphous to crystalline transition. Although there are many assumptions in their model, Capria et al. (2000) show that $\mathrm{CO}$ production rates strong enough to explain the dust coma, and comparable to or even larger than our upper limits, can be obtained with this mechanism. The activity could also be explained by the outgassing of $\mathrm{CO}_{2}$ ice (Capria et al. 2000), for which no significant devolatilization is expected in the Kuiper Belt due to its relatively low volatility (De Sanctis et al. 2001). But, this would require Chiron to have moved on its present orbit recently. The model developed for Pholus predicts $\mathrm{CO}$ fluxes lower than for Chiron because of its more distant orbit (De Sanctis et al. 2000), which might partly explain its inactive appearance.

Finally, it is interesting to note that the comparison between the Hale-Bopp $\mathrm{CO}$ outgassing rates measured at large distances from the Sun and the upper limits obtained for Centaurs and KBOs demonstrates that, indeed, these latter underwent significant CO-depletion since their formation, when compared to Oort cloud comets. A CO production rate varying approximately in $r_{\mathrm{h}}^{-2}$ and equal to $\sim 10^{28} \mathrm{~mol} \mathrm{~s}^{-1}$ at $r_{\mathrm{h}} \sim 9$ AU was measured in comet HaleBopp post-perihelion (Biver et al. 1999c). If we apply a scaling law in $r_{\mathrm{h}}^{-2} \times D^{2}$, then the $\mathrm{CO}$ production rates we infer for the corresponding $\left(D, r_{\mathrm{h}}\right)$ of our objects are, in most cases, significantly larger than the measured upper limits (Table 6).

\section{Conclusion}

We performed a sensitive search for $\mathrm{CO}$ in the Pluto/Charon system and obtained a tentative detection, which requires further confirmation, and which we interpret as an upper limit. Assuming that Charon does not contribute to the $\mathrm{CO}$ emission, these observations significantly improve published upper limits on the $\mathrm{CO} / \mathrm{N}_{2}$ mixing ratio in Pluto's atmosphere, but remain relatively unconstraining given theoretical expectations. In spite of this somewhat frustrating result, our models suggest that a mere factor-of-two improvement in instrumental sensitivity would bring much stronger constraints, if not a firm detection. The situation is similar at near-infrared wavelengths, where the $\mathrm{CO}$ absorption lines are narrow and saturated. In the millimeter range, our results are probably close to the limit achievable with presently available instrumentation, and further progress may have to await for the completion of the Atacama Large Millimeter Array (ALMA).

A more stringent upper limit was obtained on the $\mathrm{CO}$ outgassing rate in Chiron with respect to previously published results. This upper limit could be easily improved in a reasonable amount of time using the current instrumentation.

Although thermal models of KBOs predict CO outgassing rates unreachable with present instrumentation and even ALMA, further sensitive attempts might be of interest, given our present poor knowledge of these planetary bodies.

Acknowledgements. We thank S. Sheppard and J. K. Davies for their help during the CSO and JCMT observations. N. Biver was supported partly by a JCMT fellowship at the University of Hawaii. This work was supported by the Programme national de planétologie de l'Institut national des sciences de l'univers (INSU) and the Centre national de la recherche scientifique (CNRS). The JCMT is operated by the Joint Astronomy Centre on behalf of the Particle Physics and Astronomy Research Council of the United Kingdom, The Netherlands Organisation for Scientific Research, and the National Research Council of Canada. The CSO is supported by the National Science Foundation grant AST 99-80846.

\section{References}

Altenhoff, W. J., Bieging, J. H., Butler, D., et al. 1999, A\&A, 348,1020

Altenhoff, W. J., Chini, R., Hein, H., et al. 1988, A\&A, 190, L15

Altenhoff, W. J., Menten, K. M., \& Bertoldi, F. 2001, A\&A, 366, L9

Altenhoff, W. J., \& Stumpff, P. 1995, A\&A, 293, L41

Barnes, P. J. 1993, AJ, 106, 2540

Barnes, P. J. 1996, in CO: Twenty-five Years of Millimeterwave Spectroscopy, IAU Symp. 170, ed. W. B. Latter, S. J. E. Radford, P. R. Jewell, J. G. Mangum, \& J. Bally (Kluwer Academic Publishers, Dordrecht), 403

Barucci, M. A., de Bergh, C., Cuby, J.-G., et al. 2000b, A\&A, 357, L53

Barucci, M. A., Romon, J., Doressoundiram, A., \& Tholen, D. J. 2000a, AJ, 120, 496

Bauer, J. M., Meech, K. J., Buie, M. W., \& Hainaut, R. 1997, BAAS, 29, 1021

Biver, N. 2001, IWCA 2 meeting, August 14-16, 1999 (Cambridge, UK, Int. Comet Quarterly), 23-2, in press

Biver, N., Bockelée-Morvan, D., Colom, P., et al. 1999a, Earth, Moon, and Planets, 78, 5

Biver, N., Bockelée-Morvan, D., Crovisier, J., et al. 1999b, AJ, 118, 1850

Biver, N., Winnberg, A., Bockelée-Morvan, D., et al. 1999c, in Asteroids, Comets, Meteors, July 26-30, 1999 (Cornell Univ.), book of abstracts, 46 
Boice, D. C., Huebner, W. F., \& Stern, S. A. 1993, in Workshop on the Activity of Distant Comets, ed. W. F. Huebner et al. (South West Research Institute), 134

Boice, D. C., Sekiguchi, T., Ukita, N., \& Hasegawa, H. 1999, IAU Circ., 7179

Brown, R. H., Cruikshank, D. P., \& Pendleton, Y. 1999, ApJ, 519, L101

Brown, M. E. 2000a, ApJ, 119, 977

Brown, M. E., Blake, G. A., \& Kessler, J. E. 2000b, ApJ, 543, L163

Brown, M. E., \& Koresko, C. 1998, ApJ, 505, L65

Buie, M. W., Tholen, D. J., \& Horne, K. 1992, Icarus, 97, 211

Bus, S. J., A'Hearn, M. F., Bowell, E., \& Stern, S. A. 2001, Icarus, 150, 94

Bus, S. J., Buie, M. W., Schleicher, D. G., et al. 1996, Icarus, 123,478

Campins, H., Telesco, C., Osip, D., et al. 1994, AJ, 108, 2318

Capria, M. T., Coradini, A., De Sanctis, M. C., \& Orosei, R. 2000, AJ, 119, 3112

Chamberlain, J. W., \& Hunten, D. M. 1987, Theory of Planetary Atmospheres, 2nd edition (New York, Academic Press)

Colmont, J.-M., \& Monnanteuil, N. 1986, J. Quant. Spectrosc. Radiat. Transfer, 35, 81

Crifo, J.-F., Rodionov, A. V., \& Bockelée-Morvan, D. 1999, Icarus, 138, 85

Crovisier, J., Biver, N., Bockelée-Morvan, D., et al. 1995, Icarus, 115, 213

Crovisier, J., Leech, K., Bockelée-Morvan, D., et al. 1997, Science, 275, 1904

Cruikshank, D. P., Roush, T. L., Bartholomew, M. J., et al. 1998, Icarus, 135, 389

Cruikshank, D. P., Roush, T. L., Moore, J. M., et al. 1997, in Pluton and Charon, ed. S. A. Stern, \& D. J. Tholen (Univ. Arizona Press, Tucson), 221

Davies, J., Spencer, J., Sykes, M., Tholen, D., \& Green, S. 1993, IAU Circ., 5698

De Sanctis, M. C., Capria, M. T., Coradini, A., \& Orosei, R. 2000, AJ, 120, 1571

De Sanctis, M. C., Capria, M. T., \& Coradini, A. 2001, AJ, 121, 2792

Douté, S., Schmitt, B., Quirico, E., et al. 1999, Icarus, 142, 421

Elliot, J. L., \& Young, L. A. 1991, Icarus, 89, 244

Festou, M. C., Gunnarsson, M., Rickman, H., Winnberg, A., \& Tancredi, G. 2001, Icarus, 150, 140

Foster, M. J., Green, S. F., \& McBride, N. 2001, Icarus, in press

Groussin, O., Peschke, S., \& Lamy, P. L. 2001, BAAS, 32, 1031

Hainaut, O. R., Delahodde, C. E., Boehnhardt, H., et al. 2000, A\&A, 356, 1076

Haruyama, J., Yamamoto, T., Mitzutani, H., \& Greenberg, J. M. 1993, JGR, 98, 15079

Hodges, R. R. 1990, Icarus, 83, 410

Jewitt, D. C., \& Kalas, P. 1998, ApJ, 499, L103
Jewitt, D. C., \& Luu, J. X. 2000, in Protostars and Planets IV, ed. V. Mannings, A. P. Boss, \& S. S. Russel (Univ. Arizona Press, Tucson), 1201

Kern, S. D., McCarthy, D. W., Buie, M. W., et al. 2000, ApJ, 542, L155

Krasnopolsky, V. A. 1999, JGR, 104, E3, 5955

Lazzaro, D., Florczak, M. A., Angeli, C. A., et al. 1997, Planet. Space Sci., 45, 1607

Lellouch, E. 1994, Icarus, 108, 255

Lellouch, E., Paubert, G., Moreno, R., \& Schmitt, B. 2000, Icarus, 147,580

Levison, H. F., \& Duncan, M. J. 1997, Icarus, 127, 13

Luu, J. X., \& Jewitt, D. C. 1996, AJ, 112, 2310

Luu, J. X., \& Jewitt, D. C. 1998, ApJ, 494, L117

Luu, J. X., Jewitt, D. C., \& Trujillo, C. 2000, ApJ, 531, L151

McBride, N., Davies, J. K., Green, S. F., \& Foster, M. J. 1999, MNRAS, 306, 79

Millis, R. L., Wasserman, L. H., Franz, O. G., et al. 1993, Icarus, 105, 282

Owen, T. C., Roush, T. L., Cruikshank, D. P., et al. 1993, Science, 261, 745

Prialnik, D., Brosch, N., \& Ianovici, D. 1995, MNRAS, 276, 1148

Prialnik, D., \& Podolak 1995, Icarus, 117, 420

Quirico, E., Douté, S., Schmitt, B., et al. 1999, Icarus, 139, 159

Rauer, H., Biver, N., Crovisier, J., et al. 1997, Planet. Space Sci., 45, 799

Semmoud-Monnanteuil, N., \& Colmont, J.-M. 1987, J. Mol. Spect., 126, 210

Senay, M. C., \& Jewitt, D. 1994, Nature, 371, 229

Stansberry, J. A., Lunine, J. I., Hubbard, W. B., Yelle, R. V., \& Hunten, D. M. 1994, Icarus, 111, 503

Strobel, D. F., Zhu, X., Summers, M. E., \& Stevens, M. H. 1996, Icarus, 120, 266

Tegler, S. C., \& Romanishin, W. 1998, Nature, 392, 49

Tegler, S. C., \& Romanishin, W. 2000, Nature, 407, 979

Trafton, L. M., Hunten, D. M., Zahnle, K. J., \& McNutt, Jr., R. L. 1997, in Pluton and Charon, ed. S. A. Stern, \& D. J. Tholen (Univ. Arizona Press, Tucson), 475

Trafton, L. M., Matson, D. L., \& Stansberry, J. A. 1998, in Solar System Ices, ed. B. Schmitt, C. de Bergh, \& M. Festou (Kluwer Academic Publishers, Dordrecht), 773

Trafton, L. M., Stern, S. A., \& Gladstone, G. R. 1988, Icarus, 74, 108

Womack, M., \& Stern, S. A. 1999, Solar Syst. Res., 33, 187, from Astron. Vestnik, 33, 216

Yelle, R. V., \& Elliot, J. L. 1997, in Pluton and Charon, ed. S. A. Stern, \& D. J. Tholen (Univ. Arizona Press, Tucson), 347

Young, L. A., Cook, J. C., Yelle, R. V., \& Young, E. F. 2001, Icarus, in press

Young, L. A., Elliott, J. L., Tokunaga, A., de Bergh, C., \& Owen, T. C. 1997, Icarus, 127, 258 